\title{
In situ Investigation of Dielectric Breakdown in Field Effect Transistors
}

\author{
C.S. Bonifacio *, A.M. Thron *, G. Bersuker ** and K. van Benthem* \\ * Department for Chemical Engineering and Materials Science, University of California, Davis, 1 \\ Shields Ave., Davis CA 95616 \\ ** International SEMATECH, 2706 Montopolis Dr., Austin TX 78741
}

Dielectric breakdown of a field-effect transistor occurs when the insulating oxide layer fails to keep the gate capacitance [1]. As a consequence, electron tunneling through the gate dielectric leads to device failure and is inevitable with thinner gate oxides due to device scaling.

Here, we report atomic and electronic structure investigations of a field effect transistor multilayer structure of the form gate electrode $/ \mathrm{HfO}_{2} / \mathrm{SiO}_{\mathrm{x}} / \mathrm{Si}$. Although electrical properties and dielectric breakdown mechanisms have been studied before [2,3], only very limited information is available about the evolution of dielectric breakdown and its nucleation sites within individual nanometric device structures. Electrical stress was applied to gate electrodes during in situ characterization of the atomic multilayer structure. Experiments were carried out with a double-tilt STM-TEM sample holder (Figure 1). The tip of the scanning tunneling microscope (STM) was used to apply a constant positive bias to the gate electrode while measuring the induced gate current $I_{\mathrm{g}}$ across the gate dielectric. Simultaneously, atomic-scale characterization of the interface structure was performed using a JEOL $2100 \mathrm{~F} / \mathrm{Cs}$ scanning transmission electron microscope.

Figure 1 shows the measured gate current $I_{\mathrm{g}}$ as a function of time. During this first experiment, the gate bias was slowly increased with time from $0 \mathrm{~V}$ at $\mathrm{t}=0 \mathrm{~s}$ to $+10 \mathrm{~V}$ at $\mathrm{t}=155 \mathrm{~s}$. The rapid onset of the gate current indicates dielectric breakdown around $+10 \mathrm{~V}$. Future experiments will entail the application of a constant gate bias and, hence, the observation of time-dependent dielectric breakdown. Figure $2 \mathrm{a}$ and $\mathrm{b}$ show TEM micrographs of the observed multilayers prior and post dielectric breakdown, respectively. The TEM provides nanometer precision positioning of the STM tip on the gate electrode of the field effect transistor (Figure 2c). We currently conduct HRTEM, aberration-corrected STEM and STEM-EELS characterization of the interface structure before, during and after dielectric breakdown.

During the presentation, we will report in situ observations of time-dependent dielectric breakdown, i.e. the evolution of the atomic and electronic interface structures under applied electrical stress.

[1] R. Waser, Nanoelectronics and Information Technology: Advanced Electronic Materials and Novel Devices. $2^{\text {nd }}$ Edition, Germany, 2005.

[2] G. Bersuker et al., IEDM Tech. Dig. (2008)

[3] X. Li et al., IEDM Tech. Dig. (2008)

[4] The authors acknowledge financial support through start-up funds from the University of California at Davis. Technical assistance by Dr. Junhang Luo of Nanofactory during the initial STM-TEM experiments is appreciated. Parts of this research were carried out at the National Center for Electron Microscopy (NCEM) at Lawrence Berkeley National Laboratory. 


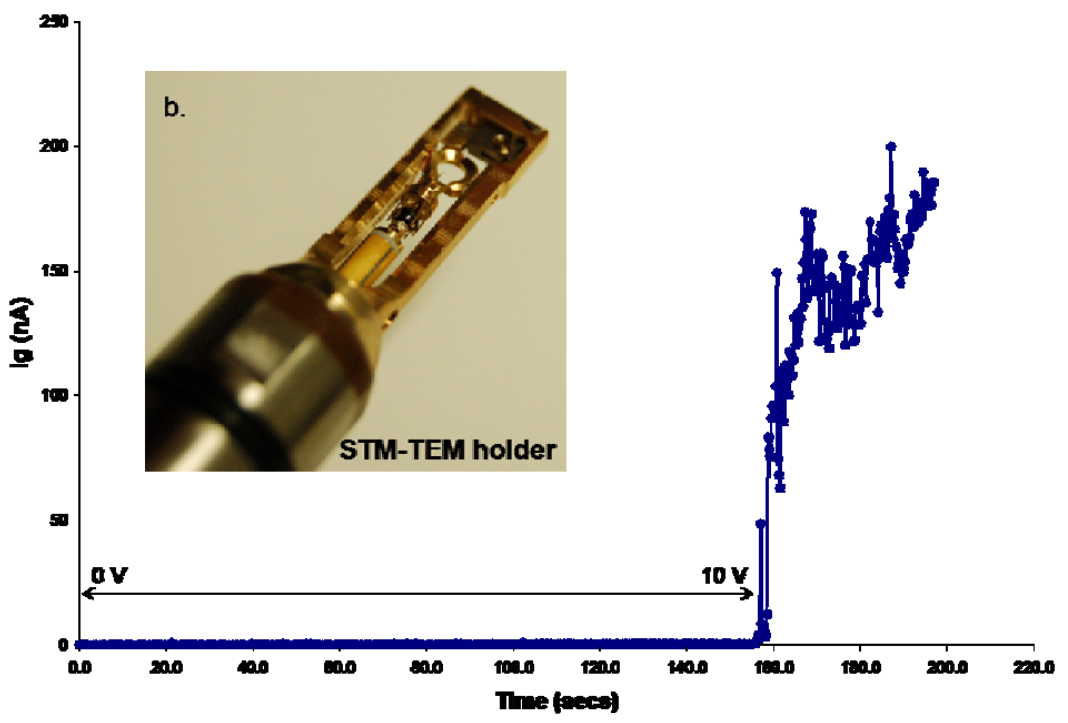

Figure 1. Evolution of dielectric oxide degradation with breakdown of the $3 \mathrm{~nm} \mathrm{HfO}_{2}$ gate oxide occurring at a gate bias of $+10 \mathrm{~V}$. Inset: image of the Nanofactory double-tilt STM-TEM specimen holder with the STM tip allowing local application of bias directly to the gate electrode.
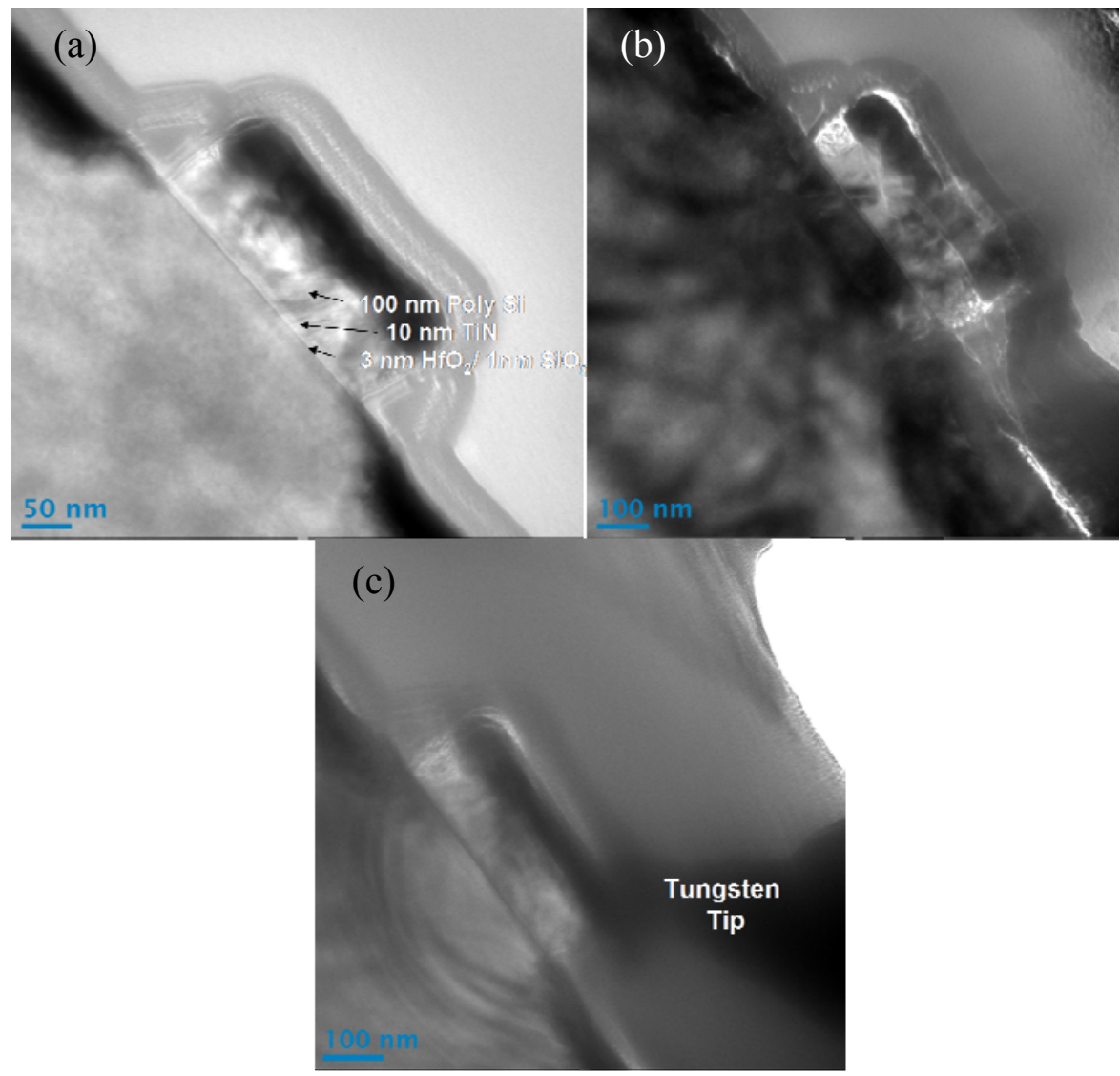

Figure 2. Cross-sectional TEM micrographs of the field effect transistor (100 nm Poly-Si and $10 \mathrm{~nm}$ $\mathrm{TiN}) / 3 \mathrm{~nm} \mathrm{HfO}_{2} / 1 \mathrm{~nm} \mathrm{SiO}$ ) before (a) and after (b) dielectric breakdown. (c) shows the STM tungsten tip precisely placed in contact to the $100 \mathrm{~nm}$ Poly-Si gate electrode. 